\section{Lymphoid flexibility}

The Pax5 transcription factor can either activate or repress transcription and is essential for B cell lineage commitment. In Immunity, Busslinger and colleagues identify 110 genes repressed by Pax 5 in pro-B cells, most of which are also expressed in other lymphoid, myeloid, erythroid and nonhematopoietic lineages. Many Pax5-repressed myeloid genes are also transcribed in common lymphoid progenitors. Induced deletion of Pax5 in pro-B cells or mature B cells results in activation of Pax5-repressed genes, demonstrating that Pax5 actively maintains the B lineage-restricted transcriptional program. During plasma cell differentiation, Pax5 expression decreases, and previously repressed Pax 5 target genes, such $\mathrm{Cd} 28$ and $\mathrm{Ccr} 2$ that are essential for plasma cell function, are reactivated. These results suggest that the lineage-specification function of Pax5 is required and must be exquisitely controlled throughout all stages of $B$ cell differentiation. $C B$ Immunity 24, 269-281 (2006)

\section{Measuring R-loops}

Immunoglobulin (Ig) class-switch recombination (CSR) exchanges the $\mu$ constant region gene segment with a downstream Ig constant region, thereby producing antibodies of differing isotype that retain antigen specificity. Ig switch (S) regions, which are repetitive and guanine rich, require transcriptional activation. Such transcripts are 'sterile', as in-frame stop codons prevent their translation, prompting questions as to the function of these RNAs in CSR. In The Proceedings of the National Academy of Science, Huang et al. show that extensive RNA-DNA hybrids (R-loops) form in $\mathrm{S}_{\gamma} 3$ and $\mathrm{S}_{\gamma} 2 \mathrm{~b}$ in lipopolysaccharide-activated splenic B cells. Rloop formation closely parallels the appearance and frequency of $\mathrm{IgG}^{+}$ switched cells. R-loop boundaries correlate with the guanine richness of the nontemplate strand, which is displaced by transcription. The R-loops thus generated might form the DNA targets of activation-induced cytidine deaminase, which is absolutely required for CSR.

$L A D$

Proc. Natl. Acad. Sci. USA 103, 5030-5035 (2006)

\section{TAP-less antigens}

Virus-infected cells and tumor cells often have defects in antigen processing and therefore have low expression of major histocompatibility complex (MHC)-peptide complexes, which allows evasion from most CD8 ${ }^{+} \mathrm{T}$ cell-mediated killing. In Nature Medicine, Offringa and colleagues demonstrate that some tumors with defects in components of the antigen-processing pathway, such as in transporter associated with antigen processing (TAP) or in the proteasome, nevertheless present peptides to $\mathrm{CD}^{+}$ T cells via a $\beta_{2}$-microglobulin-dependent mechanism. In vitro stimulation of polyclonal $T$ cells isolated from mice vaccinated with TAP-deficient tumor cells produces $\mathrm{CD} 8^{+} \mathrm{T}$ cells specific for $\beta_{2}$-microglobulin-dependent 'neoantigens'. These neoantigens obtain access to 'empty' MHC class la and Ib molecules in TAPdeficient cells, which are occupied with conventionally processed peptides in wild-type cells. Vaccination with one such neoantigen protects against challenge with TAP-deficient tumors. Enhancing MHC presentation of neoantigens may thus effectively stimulate $\mathrm{CD}^{+} \mathrm{T}$ cell responses against some tumors.

$D C B$

Nat. Med. (19 March 2006) doi:10.1038/nm1381

Research notes written by Christine Borowski, Douglas C. Braaten and Laurie A. Dempsey.

\section{New complement receptor}

Kupffer cells in the liver are important in clearing complementcoated particulate antigens from the blood. Yet the complement receptor responsible for such clearance in the liver has remained unknown. In Cell, Helmy et al. identify CRIg as the key complement receptor expressed by Kupffer cells that recognizes and removes C3opsonized particles that percolate through the liver. CRIg specifically binds to $\mathrm{C} 3 \mathrm{~b}$ or inactivated $\mathrm{C} 3 \mathrm{~b}$ fragments that are generated after C3 activation. CRIg with or without bound cargo constitutively recycles between early endosomes and the plasma membrane. Mice that lack CRIg show heightened sensitivity to blood-borne bacterial infections because of defective liver uptake of opsonized bacteria and increases in bacterial load in other organs and tissues. These results suggest that other complement or antibody receptors cannot replace the function of Kupffer cell expression of CRIg in removing C3b complexes from the bloodstream.

Cell 124, 915-927 (2006)

\section{Ineffective memory}

The function of CD40 costimulation in memory $T$ cell activation in vivo remains undefined. In the Journal of Experimental Medicine, Gray and colleagues track endogenous CD4 ${ }^{+}$ T cell function in vivo using MHC class II-peptide tetramers. Peptide immunization of wild-type but not CD40-deficient mice results in peptide-specific CD4+ $\mathrm{T}$ cell proliferation, indicating that CD40-CD40 ligand interactions are required for naive $\mathrm{CD}^{+} \mathrm{T}$ cell priming. Provision of CD40-CD40 ligand signals exclusively during priming restores peptide-specific $\mathrm{CD}^{+} \mathrm{T}$ cell proliferation and long-term survival, suggesting that $C D 4^{+}$memory $T$ cells can persist in an otherwise CD40deficient environment. However, wild-type but not CD40deficient memory $\mathrm{CD}^{+}{ }^{+} \mathrm{T}$ cells produce abundant interferon- $\gamma$ after peptide restimulation in vitro. Interferon- $\gamma$ production is restored by stimulation with peptide-pulsed, activated wildtype dendritic cells. These results demonstrate that CD40CD40 ligand signaling is essential for robust reactivation of CD4+ memory T cells.

J. Exp. Med. (20 March 2006) doi:10.1084/jem.20050711

\section{Inactivating egress}

Lymphocytes must be retained in specific lymphoid niches, such as lymph nodes, for effective immune responses to develop. In Nature, Shiow et al. demonstrate a functional link between the $\mathrm{G}$ proteincoupled sphingosine 1-phosphate type 1 receptor $\left(\mathrm{S}_{1} \mathrm{P}_{1}\right)$, required for egress of lymphocytes from lymphoid tissues, and CD69, a surface marker of lymphocyte activation and lymph node retention. Interferon$\alpha / \beta$ signaling causes concomitant upregulation of CD69 and downregulation of $\mathrm{S}_{1} \mathrm{P}_{1}$, resulting in lymphocyte retention in lymphoid tissues. In contrast, CD69-deficient lymphocytes do not lose $\mathrm{S}_{1} \mathrm{P}_{1}$ expression and are poorly retained in lymphoid tissues. Biochemical studies show that $\mathrm{S}_{1} \mathrm{P}_{1}$ and $\mathrm{CD} 69$ form a complex, and treatment of lymphocytes with pertussin toxin increases surface CD69 expression, implicating G protein-coupled-dependent signaling in the downmodulation of CD69. This previously unknown mode of lymphocyte trafficking links lymphocyte activation by interferon- $\alpha / \beta$ signaling and downregulation of a receptor required for lymphocyte egress. $\quad D C B$ Nature 440, 545-550 (2006) 